ISSN 2007-8250 / Pág. 108-117

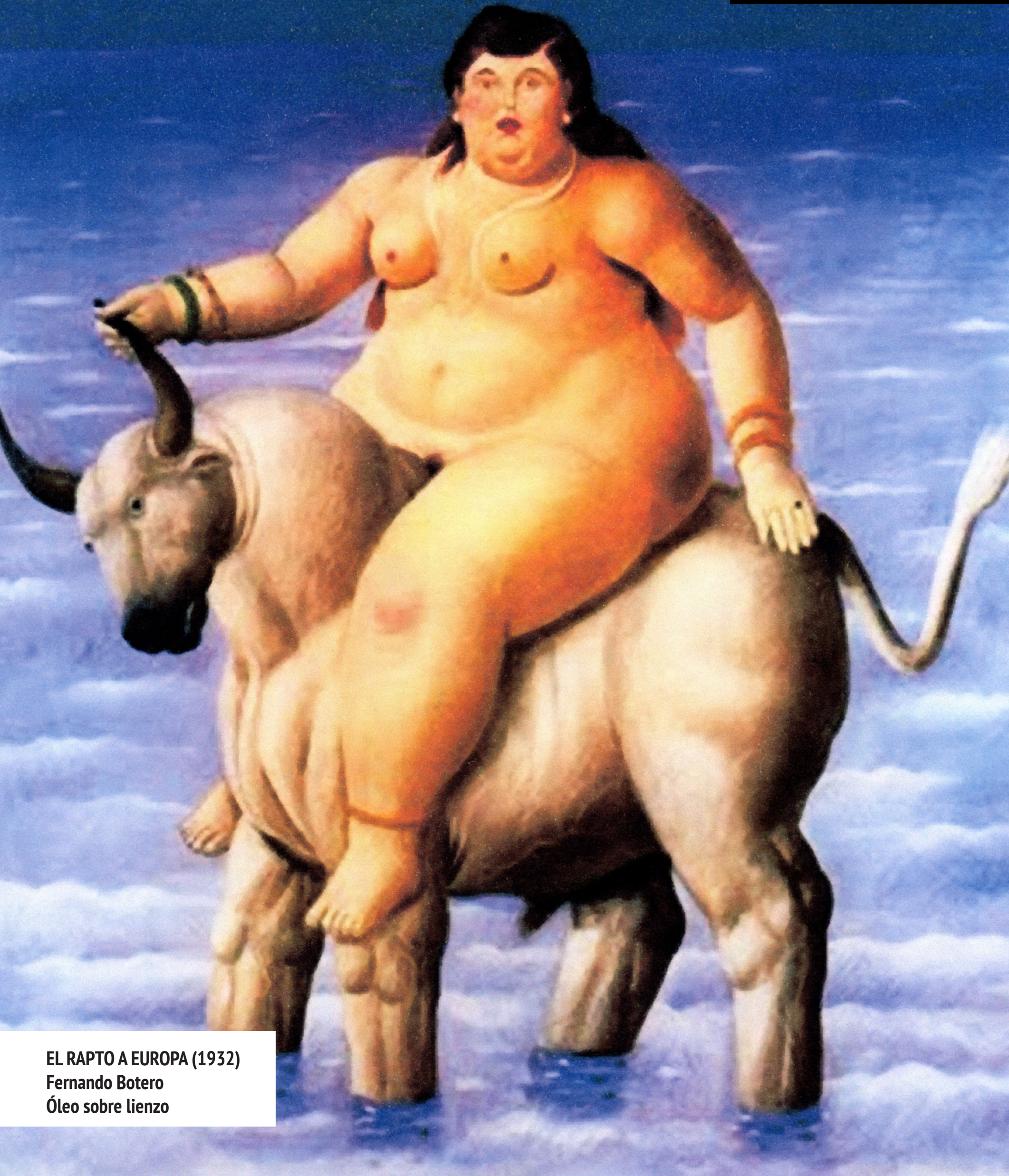




\title{
SEXO, GÉNERO Y POLÍTICA EN LA OBRA DE JEAN JACQUES ROUSSEAU Y POLULAIN DE LA BARRE
}

\author{
SEX, GENDER AND POLITICS IN THE LITERARY WORK \\ OF JEAN JACQUES ROUSSEAU AND POLULAIN DE LA BARRE
}

\section{ARTEMISA FLORES ESPÍNOLA}

\section{RESUMEN}

La importancia de los escritos y aportes de Jean Jacques Rousseau para las ciencias de la educación, la ciencia política o la sociología, es indiscutible. En este artículo, me propongo realizar una relectura de ciertos escritos realizados por Rousseau para justificar una pretendida diferencia natural entre las mujeres y los hombres y de Poulain de la Barre, un autor contemporáneo suyo que propone una concepción completamente diferente sobre la feminidad y de las mujeres. El objetivo de este trabajo fue mostrar como uno de los autores ilustrados más importantes en elaborar un discurso sobre la igualdad, puede al mismo tiempo, teorizar una filosofía política patriarcal al proponer un concepto determinado de feminidad. Este texto cuestiona el poco interés que ha suscitado la obra de Paulain de la Barre fuera de los espacios feministas.

PALABRAS CLAVE: Feminidad - Igualdad - Sociología - Patriarcado - Ciencia - Política.
ABSTRACT

The importance of the writings and contributions of Jean Jacques Rousseau for the sciences of education, political science or sociology, is indisputable. In this article, I propose to carry out a rereading of certain writings made by Rousseau to justify an alleged natural difference between women and men and of Poulain de la Barre, a contemporary author of his and who proposes a completely different conception of femininity and of women. The objective of this work is to show how one of the most important illustrated authors in elaborating a discourse on equality can, at the same time, theorize a patriarchal political philosophy by proposing a specific concept of femininity. This text questions the little interest that Paulain de la Barre's work has aroused outside of feminist spaces.

KEYWORDS: Femininity - Equality - Sociology - Patriarchy Science - Politics. 
E n su libro Fundamentos del Patriarcado Moderno. Jean Jacques Rousseau, Rosa Cobo propone un análisis de las premisas y la resignificación del patriarcado en la época de la llustración. Desde mi punto de vista, la importancia de estudiar el discurso patriarcal, principalmente el ilustrado, radica, en que estos autores legaron un nuevo paradigma a la sociedad de su tiempo y por ello continúan siendo debatidos actualmente en los espacios académicos. Sin embargo, aunque sus discursos puedan ser motivo de críticas, no es usual que se use la teoría crítica feminista para demostrar las deficiencias 0 inconsistencias de sus escritos. Con frecuencia se hace caso omiso de las pequeñas incoherencias que constituyen elaborar un discurso sobre la igualdad para todos los hombres y justificar al mismo tiempo que las mujeres no son iguales. Parece primordial evidenciar la pertinencia de la crítica feminista para identificar los sesgos en las prácticas científicas.

Rousseau no ha sido el único autor en elaborar concepciones sexistas y androcéntricas sobre los sexos. Son muchos los que han sido objeto de análisis en diversos periodos históricos y en diversas disciplinas. Tendrían que difundirse igualmente los trabajos elaborados desde una perspectiva feminista para comprender mejor los sesgos vinculados al sexo, la raza o la clase social de la persona que realiza el trabajo científico.

Los autores ilustrados ofrecieron los elementos para desmontar lo que había sido hasta entonces el entramado social y cultural del Antiguo Régimen. Con el Antiguo Régimen el orden social se basaba en el parentesco, la tierra, la religión, la monarquía, así

Rousseau no ha sido el único autor en elaborar concepciones sexistas y androcéntricas sobre los sexos. Son muchos los que han sido objeto de análisis en diversos periodos históricos y en diversas disciplinas

como en la pertenencia a una orden, una corporación, una comunidad local. Al proclamar la igualdad jurídica entre los ciudadanos, la ilustración y después la revolución francesa cuestionó los fundamentos mismos del orden político tradicional, que se basaba en la jerarquía y la desigualdad de estatus. La sociedad del Antiguo Régimen estaba dividida en tres órdenes desiguales y jerárquicas. El discurso de los ilustrados consistía en liberar al individuo de las limitaciones sociales heredadas del pasado. En lugar de reproducir la sociedad legada por nuestros antepasados, en particular renovando sus viejas jerarquías y tradiciones, los ilustrados proclamaban la igualdad entre los ciudadanos. Sin embargo, hay que entender, por qué las mujeres no eran consideradas ciudadanas. Estas son algunas de las respuestas de dos famosos filósofos ilustrados.

Iniciaré con uno de los pensadores más famosos de la ilustración: Jean Jacques Rousseau (1712-1778). Rousseau fue huérfano de madre y su padre lo abandonó a los 10 años. Por lo que se convierte en un autodidacta. Estos hechos de su vida, marcarían el camino que habrían de llevar sus obras. Entre sus obras más importantes se encuentran El Emilio y Discurso sobre el origen y los fundamentos de la desigualdad entre los hombres. Nos concentramos en sus premisas más importantes para justificar una soberanía de los hombres en lo público y una soberanía de las mujeres en lo privado. Rousseau definirá 2 roles para los hombres y para las mujeres, funciones complementarias, en los que las mujeres deberán ayudar y servir a los hombres a que sean libres y autónomos. Él plantea una división sexual del trabajo, no dejando posibilidades para las mujeres de poder compartir el mundo de lo público. Sino exponiendo las razones por la cuáles deben permanecer en casa. Por este motivo -de acuerdo con las interpretaciones de Rosa Cobo- puede ser considerado como el fundador del patriarcado moderno ${ }^{1}$.

Para Rousseau las diferencias entre niños y niñas determinan su educación. Por tanto, la educación de las niñas deberá de prepararlas para la abne-

${ }^{1}$ Cfr. Rosa Cobo (1995). Fundamentos del patriarcado moderno. Jean Jacob Rousseau. Col. Feminismos. Madrid: Cátedra 
gación, la renuncia y la sumisión a los deseos de los otros. Si la educación no lograba esto, probablemente la mujer sufriría en el futuro para adaptarse a las instituciones (particularmente el matrimonio) en las cuales debía de desarrollar su vida. Rousseau indica:

Las niñas deben ser activas y diligentes, pero eso no es todo; desde muy temprano han de ser propensas a saber contenerse. Esta desventura, en caso de que lo sea realmente, es inseparable de su sexo, y nunca se han librado de ella sino para soportar infortunios mayores. Deben someterse al decoro durante toda su vida, que es el freno más severo y constante. Es necesario, por tanto, acostumbrarlas desde pequeñas a esta limitación, no sea que después les cueste demasiado caro. En lo que respecta a la supresión de caprichos, deben estar dispuestas a aceptar el deseo de los demás. Si, por ejemplo, les gusta estar siempre trabajando, hay que impulsarlas a que lo dejen de vez en cuando. Demasiada indulgencia las corrompe y pervierte con la di-

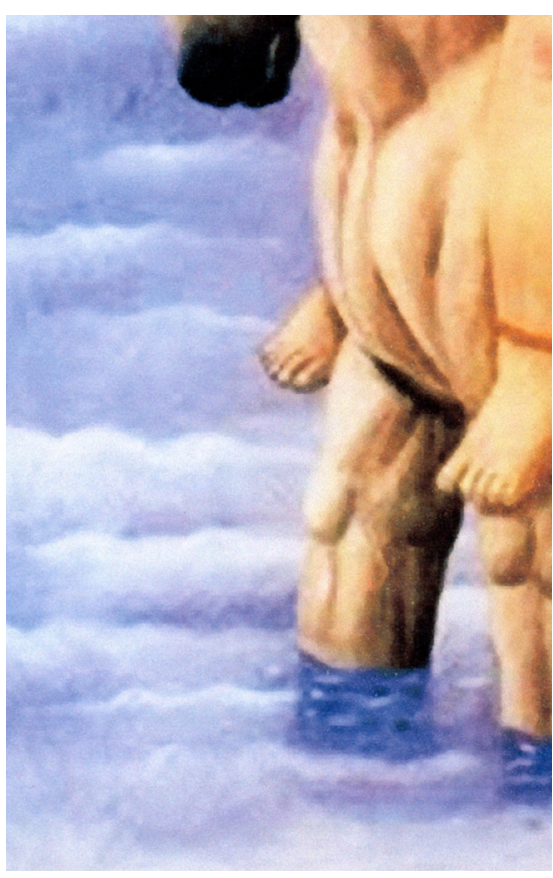

sipación, la vanidad y la inconstancia, que son los vicios a los que son más propensas. Para prevenir estos abusos debemos enseñarlas ante todo, a saber mantenerse en los límites debidos. La vida de una mujer modesta se reduce, debido a todas nuestras instituciones absurdas, a un perpetuo conflicto consigo misma. Pero esto es justamente porque de alguna manera deben participar en los sufrimientos que su sexo demoníaco nos ha causado².

Como vemos, la idea de Rousseau, es que la mujer cumpla con lo que la feminidad normativa establece. Pero habría que hacer una puntualización, si Rousseau piensa que hay que enseñar a las mujeres, es porque se da cuenta que lo que se pide de ellas, no es algo que venga dado con la naturaleza. El habla de un modelo para construir a las mujeres sumisas y abnegadas, lo cual será sin duda fundamental para que exista el ciudadano libre. Su misoginia y sus intereses, en tanto que hombre, le hacen negarle los derechos que él mismo defendió para todos los

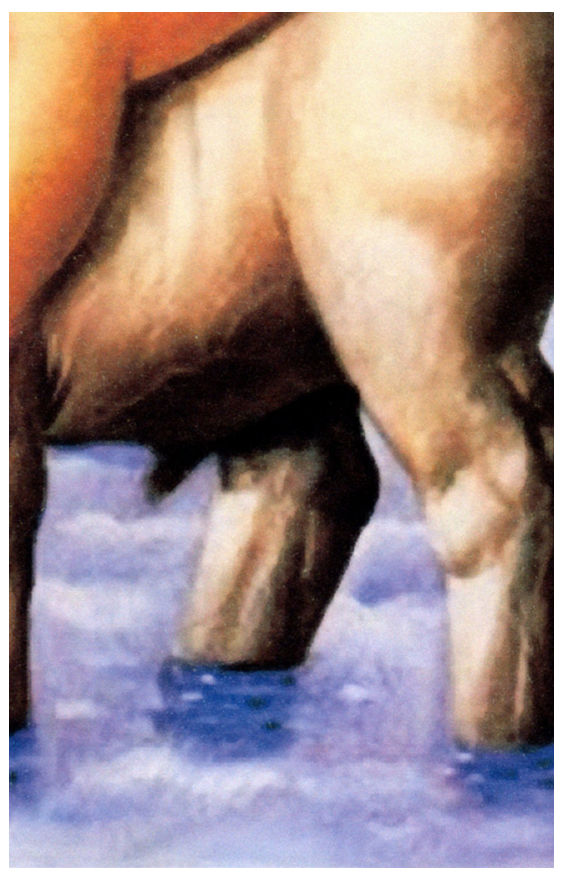

hombres como la ciudadanía y colaborar del contrato social ${ }^{3}$.

Rousseau desarrolló una conceptualización de las mujeres como inferiores a los hombres y con un rol social y familiar determinado por el varón. Nada nuevo. Para Rousseau los niños a los colegios, las mujeres a los conventos. Lo que hará Rousseau es radicalizar precisamente las diferencias entre los sexos. En su clásica novela El Emilio, en el capítulo V, dibuja a una mujer, Sofía, como carente de cualidades y habilidades naturales para el conocimiento y el desarrollo de roles de la esfera pública. Centra a la mujer en la esfera privada, en el cuidado de los hijos, la atención a los trabajos domésticos y le ofrece como posibilidad el colaborar en el desarrollo del hombre liberándose de las cargas domésticas para que se dedique enteramente a la vida pública. Como muy bien sintetiza Cristina Molina:

Sin la Sofía doméstica y servil, no podría existir el Emilio libre y autónomo.

Es evidente que en general, en la época ilustrada, la mujer permanecía totalmente en el ámbito privado, considerándose a su vez, dentro de

2Jean Jacob Rousseau (1979). Discurso sobre el origen y los fundamentos de la desigualdad entre los hombres. Citado en: Escritos de Combate. Tr. Y notas de Salustiano Masó. Madrid: Alfaguara. pp. 137-138.

${ }^{3}$ Sin entrar en detalles, también diremos que Rousseau tampoco consideraba iguales a todos los hombres como colectivo."La inteligencia de los europeos no la tienen, los negros ni los lapones. Por eso, si quiero que mi alumno pueda ser habitante de la tierra entera, le escogeré en una zona templada, en Francia, por ejemplo, mejor que en otra parte". En: $E l$ Emilio. pág. 32. 
este ámbito todo lo concerniente a sus necesidades y su satisfacción. La idea rousseauniana de que la mujer pertenecía al ámbito privado y que su educación le debería ayudar para tal efecto, corresponde a un prejuicio sexista. Los prejuicios sexistas fueron desarrollados ampliamente en la obra del filósofo francés Poulain de la Barre. Rousseau escribe en el capítulo V de su libro El Emilio que la desigualdad de las mujeres no es obra del prejuicio, sino de la razón. Lo cual, no deja de ser curioso porque es la tesis defendida por Poulain de la Barre ${ }^{4}$ en su libro De l'égalité des deux sexes. Algunas autoras como Fraisse o Amorós señalan que existen indicios para pensar que Rousseau conocía la obra de este cartesiano francés. La obra de Poulain de la Barre ha sido bastante difundida en Francia por la filósofa Geneviève Fraisse y en España por Celia Amorós 5 . No solamente en sus escritos sino también recurrentemente analizados en los cursos universitarios. Reconocer que las desigualdades se encuentran en la fuerza de la costumbre y no en la naturaleza, es un argumento importante utilizado no solamente por las teorías construccionistas en ciencias sociales. Basándose en esta idea de las costumbres y del prejuicio en la base de la desigualdad de los sexos, será lo que, tiempo después Poulain de la Barre desarrollará y justificará teóricamente

Otra idea importante que tiene ser analizada en la obra de Rousseau es lo concerniente al contrato social. Ya que las mujeres no participan de este acto en la sociedad. Debido a que lo político surge del contrato social, pero como la desigualdad es natural, entonces no se les reconoce como sujetos y por tanto no pueden ser ciu-

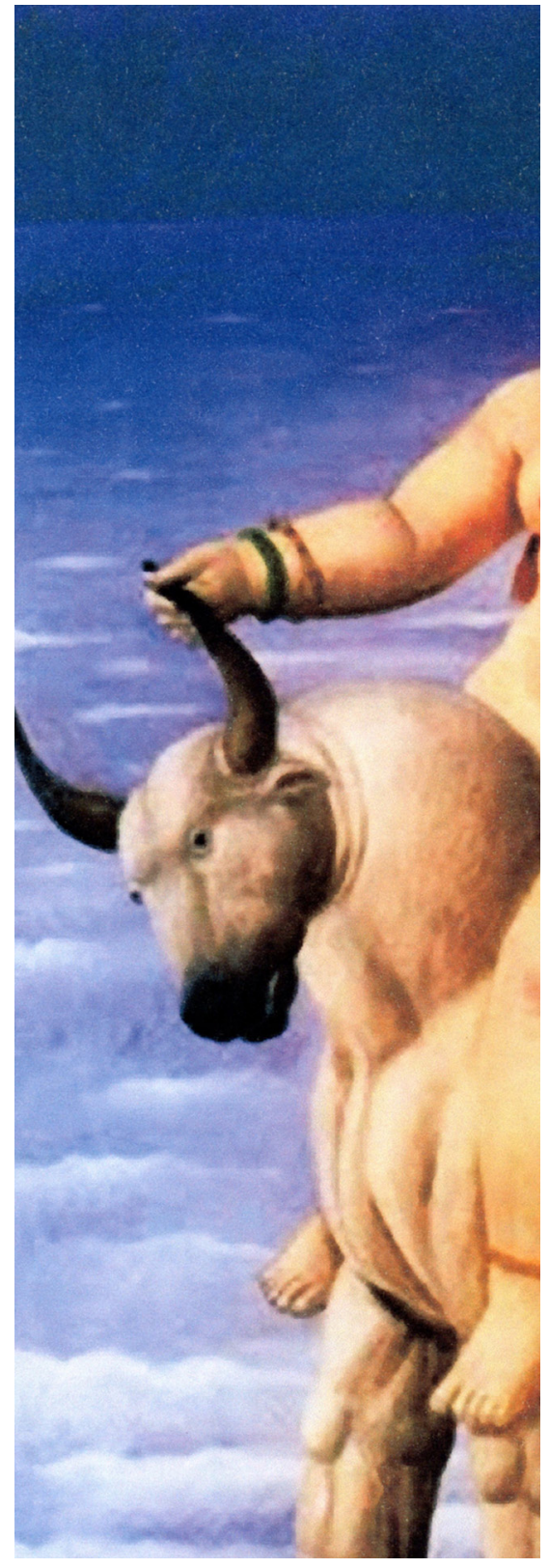

dadanas. De esta manera, se asegura que no participe en el espacio que no le corresponde como es el público y se la determina en el espacio privado como reproductora del sistema social y la encargada de la educación primaria de los futuros Emilios. De esta manera sus planteamientos de igualdad se establecen sólo entre los hombres y no entre hombres y mujeres. Porque para Rousseau todas las diferencias entre hombres y mujeres son debidas al sexo. Para este autor, las hembras están atadas a su sexo toda su vida y los machos sólo unos instantes. En palabras de Celia Amorós:

...En el varón, la relación es meramente puntual y accidental, mientras que en la mujer es esencial: la una se define por su diferencia sexual y el otro no. De nuevo, el masculino se solapa con el neutro...y

${ }^{4} \mathrm{Cfr}$. C. Amorós. Tiempo de Feminismo. Op. Cit. Amorós realiza un seguimiento sobre ciertos trabajos de investigación en que se contratastan los textos de ambos autores. La tesis que intentan probar, es que Rousseau pudo haber tenido acceso a la obra de Poulain de la Barre, pero no aparece citado en sus trabajos.

${ }^{5}$ Cf. Geneviève Fraisse (1991). Musa de la razón. Cátedra. Madrid. 


\section{Como dijo Montesquieu:}

\section{Las fuerzas serían iguales si la educación \\ lo fuera también}

asume lo genéricamente humano, que, en el discurso ilustrado, se define por la universalidad, por la igualdad de todos los sujetos, que lo son en la medida en que no están sujetos al sexo, como las mujeres...6

Pero Rousseau, aunque influyente y determinante para la visión de su época, no fue el único que nutrió con sus ideas el discurso ilustrado que favorecía la desigualdad entre hombres y mujeres.

A mediados de este siglo XVII surge una figura masculina que será sin duda muy importante para el feminismo: Poulain de la Barre (1647-1723). Doctor en Teología y ordenado sacerdote. Sin embargo, será considerado un libre pensador, una persona escéptica. Se convierte al protestantismo, se casa y se va a vivir a Ginebra. Su libro De l'égalité des deux sexes, a pesar de haber sido escrito en su juventud, resulta sólidamente fundamentado y se presenta como un estudio de la construcción de la identidad social de la mujer. La importancia de este autor ha sido señalada por diversos trabajos y como mencionaba Beauvoir:

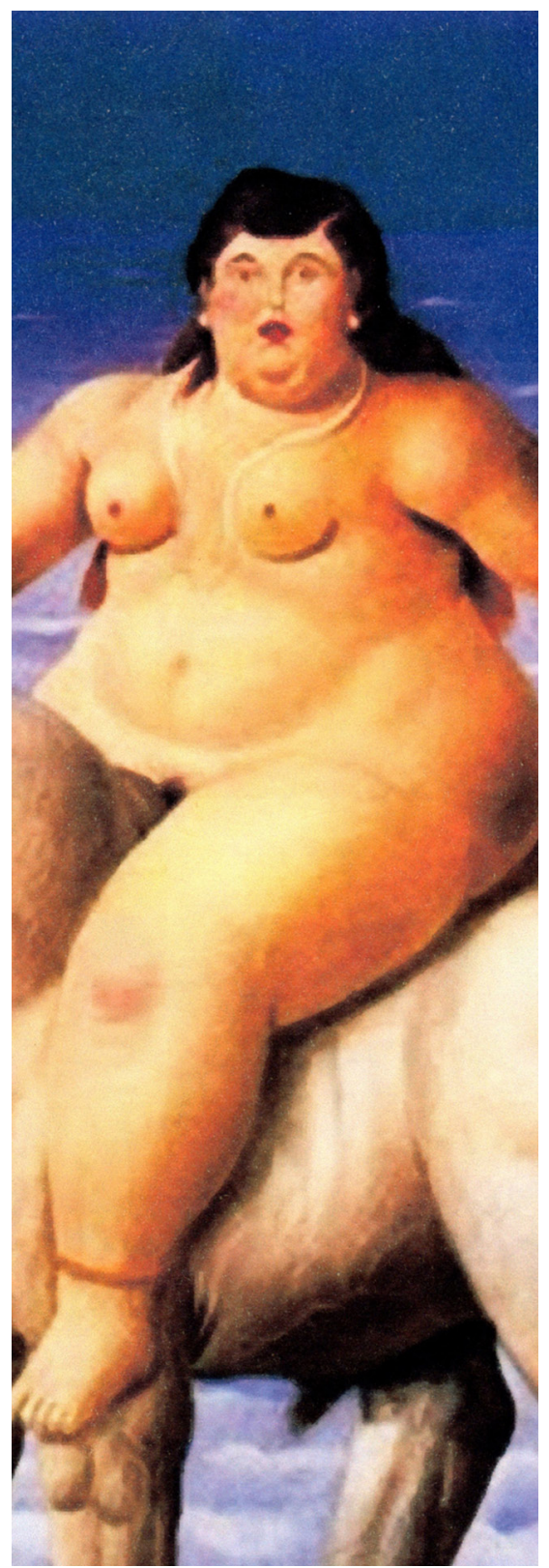

El feminista más resuelto de la época es Poulain de la Barre, que publica en 1673 una obra de inspiración cartesiana, De l'égalite des deux sexes. Considera que como los hombres son más fuertes siempre han tratado de aventajar a su sexo y que las mujeres aceptan por costumbre esta dependencia. Nunca tuvieron una oportunidad, ni libertad, ni instrucción. No se las puede juzgar por lo que han hecho en el pasado. Nada indica que sean inferiores al hombre. La anatomía refleja diferencias, pero ninguna es privilegio del varón. Poulain concluye reclamando para las mujeres una sólida instrucción.

Esto es algo esencial y que también maneja Beauvoir en sus escritos. Las mujeres hasta el siglo XVII no han tenido oportunidad para estudiar y una cierta libertad. Lo cual las ha convertido en seres dependientes material y jurídicamente. Pero debemos subrayar, esto es un hecho histórico y no una decisión personal. Poulain además de combatir porque a las mujeres se les deje acceder al conocimiento, también reivindica una justicia social para todos y todas. Como dijo Montesquieu:

Las fuerzas serían iguales si la educación lo fuera también.

Ya bien lo señalaba Geneviève Fraisse, que con Poulain de la Barre se cambia la recurrente controversia entre los sexos, ya que el autor no compara a los sexos, sino que va más allá reflexionando sobre la igualdad.

Para Celia Amorós, el primer texto vindicativo se puede establecer en el siglo XVII con Poulaine de la Barre. Este sacerdote que escribió libros sobre

${ }^{6}$ C. Amorós (1997). Op. Cit., pág. 160. 
teoría feminista. Era un autor racionalista seguidor de Descartes. Su teoría gira en torno a aplicar la duda metódica al campo del conocimiento, en no fiarnos de la tradición, ni de las sagradas escrituras, en aplicar la sospecha a la epistemología. Poulaine tiene una concepción del prejuicio menos racionalista que Descartes, en donde lo que hace es exprimir las virtualidades del método cartesiano para las reformas de las costumbres.

Las mujeres están ellas mismas tan convencidas de su desigualdad y de su incapacidad, que no sólo convierten en virtud soportar la dependencia a la que están sometidas, sino incluso creen que ésta se funda en la diferencia que la naturaleza ha dispuesto entre ellas y los hombres. Recuerda muy buen que cuando el libro de la Igualdad apareció, sólo las preciosas lo recibieron con entusiasmo al decir que les hacía alguna justicia, otros le dieron valor sobre todo porque halagaba su vanidad; pero el resto habló de él como una paradoja que tenía más de galantería que de verdad...

En esta referencia vemos la idea que tenía Poulain sobre las preciosas y el efecto que su primer libro sobre $L a$ igualdad de los sexos (1673), había creado en el momento circunstancial de su divulgación entre las mujeres. Sin duda es de asombrarse que las preciosas no hubieran celebrado y tal vez querido continuar con su obra. Pero como ya dijimos, las preciosas eran grupos de mujeres intelectuales no necesariamente feministas. Por eso Poulain de la Barre sería la primera persona en fundamentar sobre teoría feminista. Poulain establece, en primer lugar, la idea de prejuicio como una

\section{El planteamiento de Poulain es cartesianamente} nítido y podría resumirse así: el prejuicio relacionado con la desigualdad de los sexos es el más obstinado y ancestral, ergo, si podemos refutarlo

forma distorsionada de ver y configurar la realidad social. En sus análisis con respecto a la situación de la mujer, establece que la desigualdad que se creía natural en esa época, en realidad obedece a un prejuicio. Poulain indica: ...entre todos los prejuicios, ninguno hemos podido observar que fuera más apto para nuestro empeño que aquél que comúnmente se tiene sobre la desigualdad de ambos sexos...

Se mostró con algunas citas de algunos pensadores misóginos que aluden siempre a las diferencias físicas entre hombres y mujeres, diciendo que esto se traduce en una desigualdad intelectual. El vislumbraba una época de oro, donde no habría una repartición sexual de tareas. Por ello, para desmentir estas ideas tan arraigadas socialmente realiza su análisis de la igualdad entre los sexos, Poulain recurre a la igualdad de espíritu que tienen tanto el hombre y la mujer, esto es la esencia de su pensamiento l'esprit n'a point de sexe decir de Celia Amorós:

El planteamiento de Poulain es cartesianamente nítido y podría resumirse así: el prejuicio relacionado con la desigualdad de los sexos es el más obstinado y ancestral, ergo, si podemos refutarlo (derivando de las premisas cartesianas que l'esprit no tiene sexo), a fortiori lo podremos hacer con todos los demás; 0 , dicho de otro modo, habremos contrastado las condiciones de posibilidad, no sólo lógicas sino pragmáticas del programa cartesiano de lucha contra el prejuicio ampliado -con connotaciones ilustradas- al ámbito de la praxis social.

Como podemos observar claramente, el primer análisis filosófico propiamente feminista es el de Poulain, el cual se basa en un racionalismo bastante eficiente y por lo tanto, diríamos, pre-ilustrado (aunque este autor no se puede considerar propiamente como ilustrado). Es en este autor donde más se puede apreciar el origen pre-ilustrado del feminismo. Tomando en cuenta la herramienta ilustrada por excelencia: la razón, y al aplicarla para analizar las diferencias entre los sexos, el autor ha llegado a la conclusión de que se tienen suficientes bases para dudar de esa verdad y por consiguiente para formular la idea de que, independientemente del sexo, 
se tiene espíritu y el espíritu es lo más importante del ser humano, por lo tanto hombre y mujer son iguales en lo esencial, que es el espíritu, de lo cual se sigue que ambos deberían operar con principios fundamentales de igualdad y no de desigualdad.

Hemos visto un poco los principios básicos de su primer libro, en donde explica cómo ha sido el prejuicio el que ha hecho que se consideren diferentes a los sexos y fundamenta la igualdad como portadoras del buen sentido cartesiano. En el segundo (del que sólo tenemos algunos pasajes, pero no el acceso completo al libro), que se tituló De l'excellence des homme,s, el autor menciona que las mujeres estarían en un nivel intelectual superior al no estar contaminadas como los hombres, serían las portadoras del buen sentido por excelencia y, por tanto, serían ellas las únicas que pueden reconducir a los hombres al buen sentido y de esa manera cambiarían de parecer de sus ideas respecto a las mujeres. Esta contradicción aparente en su segundo libro, podríamos resumirlo con estas palabras de Amorós:

De este modo, en la medida en que le bon sens se contrapone radical- mente como instancia crítica al saber tradicional instituido, y dado que son sobre todo las mujeres las que tradicionalmente han sido excluidas de ese saber... resultan ser ellas las menos corrompidas, precisamente en virtud de que su propia marginación les ha ahorrado el farragoso bagaje de estratos de saber acríticamente asumidos, y están así mejor preparadas que nadie para ejercer le bon sens con toda soltura y menos hipotecas.

Se puede estar de acuerdo o no con las ideas de Poulain de la Barre o de Rousseau. Cada persona puede hacer uso de sus conocimientos para elegir entre estas dos propuestas opuestas, pero es importante reconocer que en esta selección entra en juego también nuestra experiencia subjetiva, nuestros prejuicios. Este artículo considera los valores propios del contexto social de la investigación científica como constitutivos de las ciencias. Es decir, lo queramos 0 no, en las prácticas científicas no solo los valores constitutivos a la ciencia (verdad, simplicidad, predictividad, consistencia, rigor y precisión), sino también los valores contextuales (sociales, culturales, mo-

\section{Cada persona puede hacer uso}

\section{de sus conocimientos para elegir entre estas}

\section{dos propuestas opuestas, pero es importante}

reconocer que en esta selección entra

en juego también nuestra experiencia

subjetiva, nuestros prejuicios

rales) influyen en la ciencia (Longino, 1999 y 2006). Desde nuestras decisiones de carrera, de temas de investigación, las teorías que adoptamos y en la interpretación de los resultados de la investigación.

Esto conlleva a reflexionar sobre la necesidad de impulsar más trabajos de investigación que muestran las contradicciones de ciertos discursos arraigados y que no conllevan a una transformación de la sociedad. No todas las ciencias buscan el cambio social, pero ciertas corrientes sociológicas y feministas asumen que, si los valores contextuales conservadores se encuentran presentes en las prácticas científicas y educativas, porque no podrían también incluirse los valores humanistas y emancipadores.

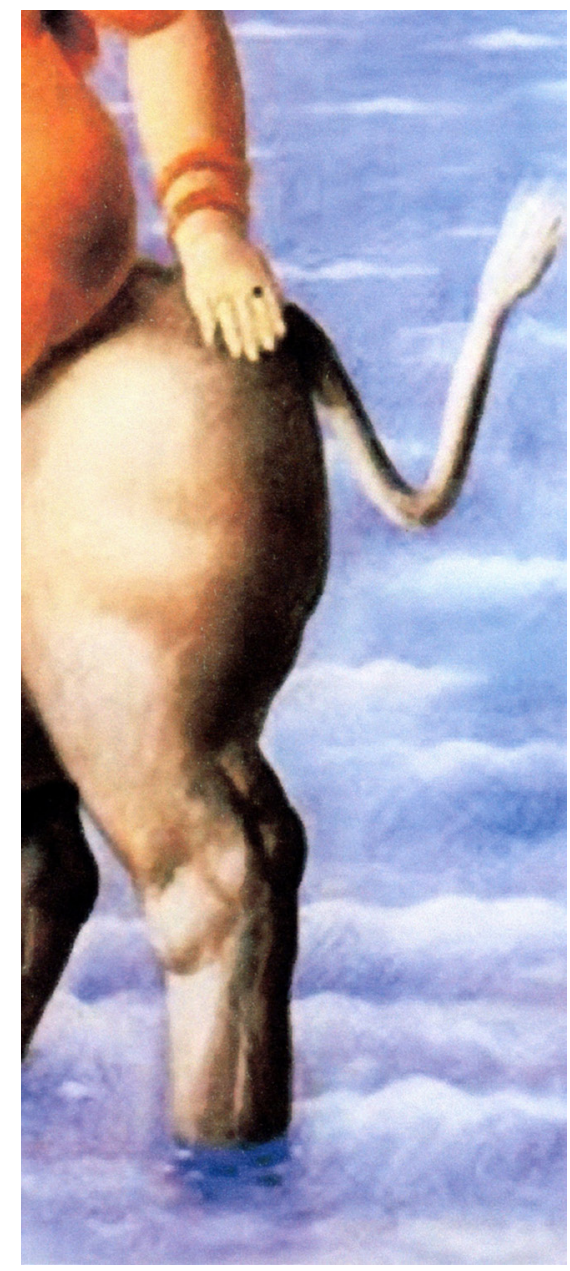




\section{REFERENCIAS}

Cfr. Rosa Cobo (1995). Fundamentos del patriarcado moderno. Jean Jacob Rousseau. Col. Feminismos. Madrid: Cátedra.

Jean Jacob Rousseau (1979). "Discurso sobre el origen y los fundamentos de la desigualdad entre los hombres". Citado en: Escritos de Combate. Tr. Y notas de Salustiano Masó. Madrid: Alfaguara. pp.137-138.

Sin entrar en detalles, también diremos que Rousseau tampoco consideraba iguales a todos los hombres como colectivo. "La inteligencia de los europeos no la tienen, los negros ni los lapones. Por eso, si quiero que mi alumno pueda ser habitante de la tierra entera, le escogeré en una zona templada, en Francia, por ejemplo, mejor que en otra parte". En: El Emilio. pág. 32.

Cfr. C. Amorós. Tiempo de Feminismo. Op. Cit. Amorós realiza un seguimiento sobre ciertos trabajos de investigación en que se contrastan los textos de ambos autores. La tesis que intentan probar, es que Rousseau pudo haber tenido acceso a la obra de Poulain de la Barre, pero no aparece citado en sus trabajos.

Cf. Geneviève Fraisse (1991). Musa de la razón. Cátedra. Madrid.

C. Amorós (1997). Op. Cit., pág. 160

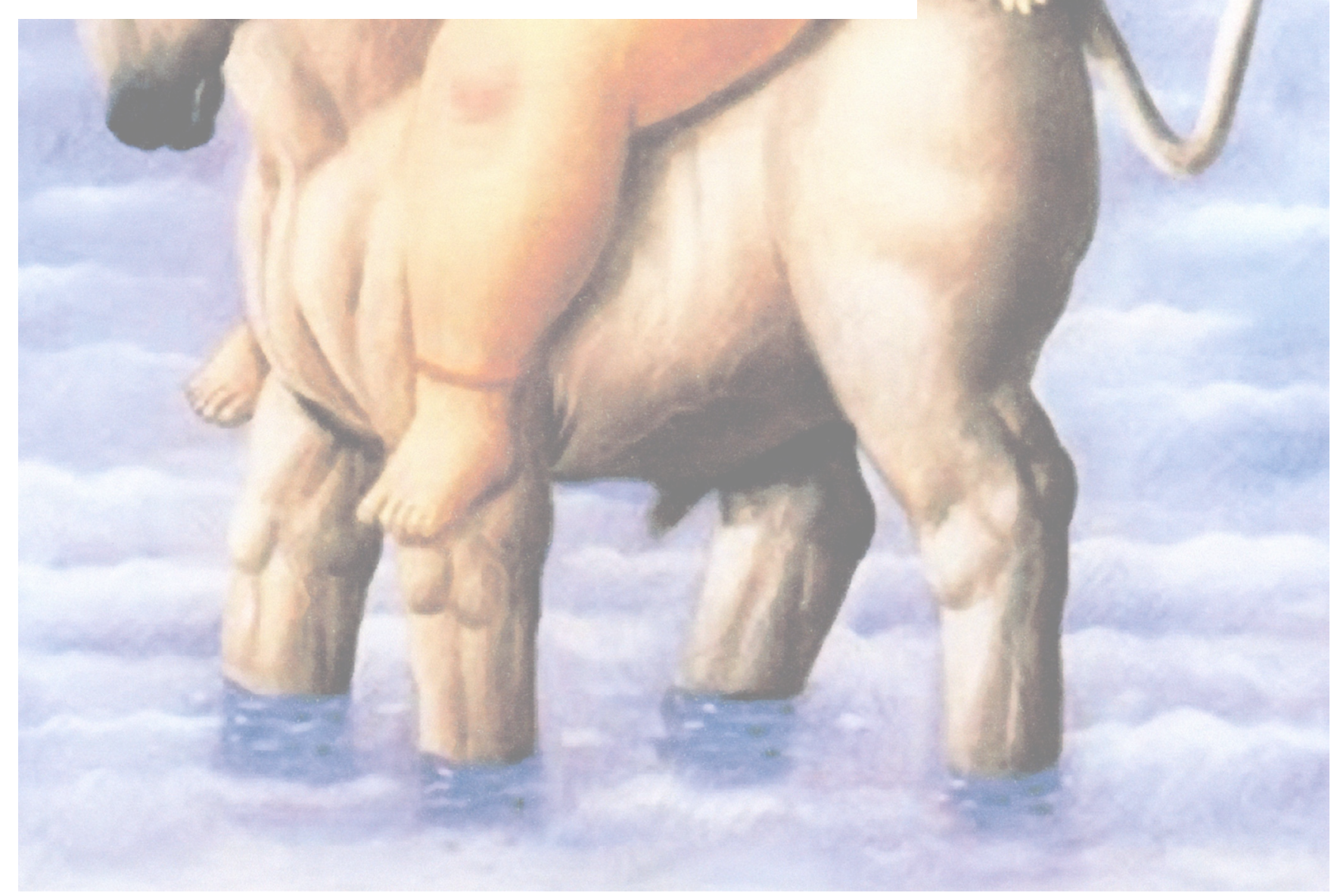




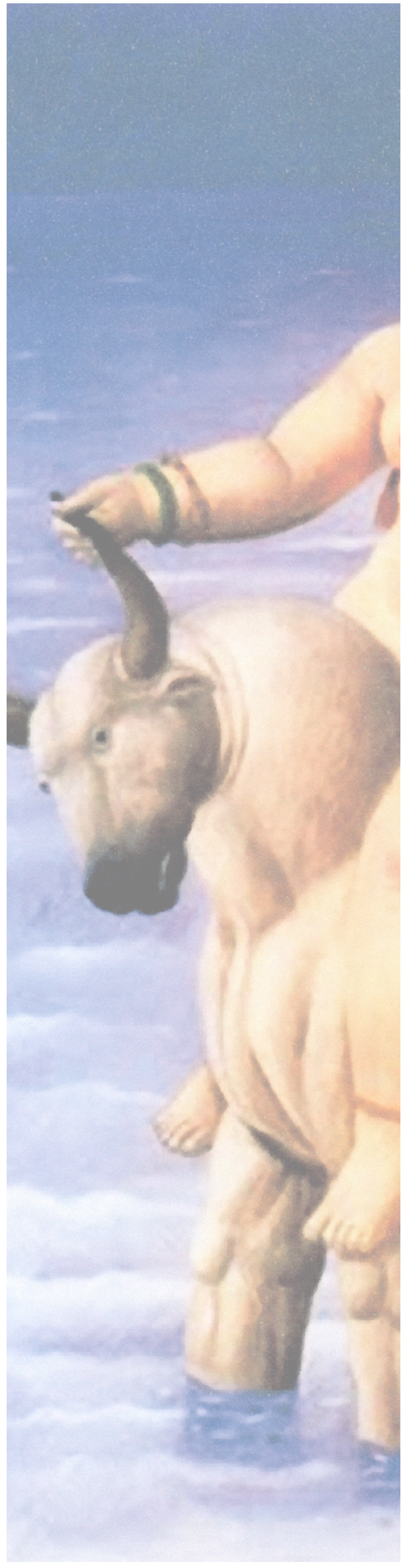

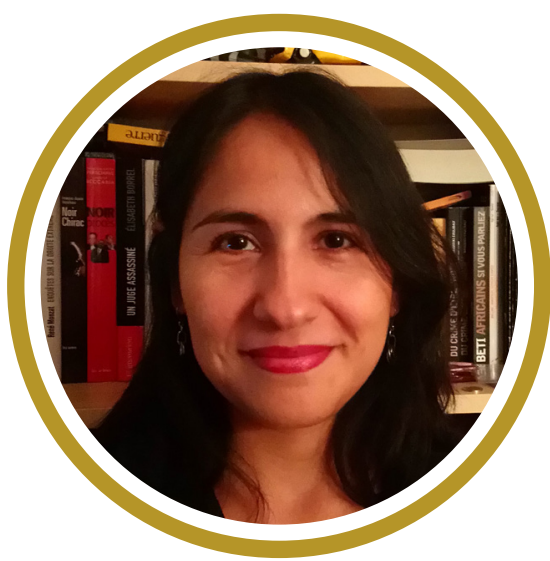

Artemisa

Flores Espínola

Licenciada en sociología por la Universidad Autónoma de Nuevo León y realizó su master en la Universidad Complutense de Madrid (UCM). Obtuvo un Doctorado Europeo en Sociología por la UCM y la Universidad de Paris. Actualmente es Profesora-Investigadora en Sociología, Universidad de Paris-Est Créteil Laboratorio Interdisciplinario de Investigación sobre las transformaciones de las prácticas educativas y de las prácticas sociales (LIRTES). Es investigadora en Centro de Investigaciones Sociológicas y Políticas de Paris (CRESPPA) del CNRS. Sus principales líneas de investigación son: género, ciencia y tecnología; metodologías y epistemologías feministas; sociología de las profesiones y del reconocimiento científico; género y educación. Desde el 2011 es responsable (con Danièle Kergoat y Jules Falquet) de la red temática "Sexo, clase y género" de la Asociación Francesa de Sociología

\section{Correo Electrónico:}

artemisa.flores@gmail.com

Fecha de Recepción: 15/03/20

Fecha de Aprobación: 15/08/20 